\title{
Printed Graphene Radio Frequency and Microwave Electronics
}

\author{
Zhirun Hu \\ The University of Manchester, UK
}

\begin{abstract}
Graphene is a two-dimensional (2D) planar layer of carbon atoms packed in a honeycomb lattice. It has been drawing a great attention from both research institutes and industry. Due to the unique chemical, thermal, mechanical, electronic and optical properties of graphene, many studies on its application for RF, microwave/millimeter-wave and THz devices and circuits have been reported. In this talk, the main focus will be on exfoliation of highly conductive graphene ink and printed graphene RF/microwave components on flexible substrates such as papers and textiles. Environmentally sustainable production of highly conductive graphene ink suitable for screen-printing technology has been developed and used for printing various low-cost, flexible, disposable antennas, transmission lines and sensors. Low-cost and environmentally sustainable printed graphene technology has a potential to penetrate to information technology into our everyday life, especially in promoting wireless connectivity, energy harvesting and the spread of IoT applications.
\end{abstract}

